\title{
Purified and Recombinant Hemopexin: Protease Activity and Effect on Neutrophil Chemotaxis
}

\author{
Tian Lin, ${ }^{1 *}$ Jialin Liu, ${ }^{2 *}$ Feng Huang, ${ }^{2 *}$ Tjitske SR van Engelen, ${ }^{2}$ Sujatha R Thundivalappil, ${ }^{2}$ Frank E Riley, ${ }^{2}$ \\ Michael Super, ${ }^{3}$ Alexander L Watters, ${ }^{3}$ Ann Smith, ${ }^{4}$ Nathan Brinkman, ${ }^{5}$ Donald E Ingber, ${ }^{3}$ and H Shaw Warren ${ }^{6}$
}

${ }^{1}$ Department of Pediatrics, Infectious Disease Unit, Massachusetts General Hospital and Harvard Medical School, Boston, Massachusetts, United States of America; ${ }^{2}$ Department of Pediatrics, Infectious Disease Unit, Massachusetts General Hospital, Boston, Massachusetts, United States of America; ${ }^{3}$ Wyss Institute at Harvard, Center for Life Science, Boston, Massachusetts, United States of America; ${ }^{4}$ Division of Molecular Biology and Biochemistry, School of Biological Sciences, University of Missouri, Kansas City, Missouri, United States of America; ${ }^{5}$ CSL Behring LLC, Research and Development, Kankakee, Illinois, United States of America; ${ }^{6}$ Department of Pediatrics and Medicine, Infectious Disease Unit, Massachusetts General Hospital, and Harvard Medical School, Boston, Massachusetts, United States of America

\begin{abstract}
Infusion of the heme-binding protein hemopexin has been proposed as a novel approach to decrease heme-induced inflammation in settings of red blood cell breakdown, but questions have been raised as to possible side effects related to protease activity and inhibition of chemotaxis. We evaluated protease activity and effects on chemotaxis of purified plasma hemopexin obtained from multiple sources as well as a novel recombinant fusion protein Fc-hemopexin. Amidolytic assay was performed to measure the protease activity of several plasma-derived hemopexin and recombinant Fc-hemopexin. Hemopexin was added to the human monocyte culture in the presence of lipopolysaccharides (LPS), and also injected into mice intravenously (i.V.) 30 min before inducing neutrophil migration via intraperitoneal (i.p.) injection of thioglycolate. Control groups received the same amount of albumin. Protease activity varied widely between hemopexins. Recombinant Fc-hemopexin bound heme, inhibited the synergy of heme with LPS on tumor necrosis factor (TNF) production from monocytes, and had minor but detectable protease activity. There was no effect of any hemopexin preparation on chemotaxis, and purified hemopexin did not alter the migration of neutrophils into the peritoneal cavity of mice. Heme and LPS synergistically induced the release of LTB4 from human monocytes, and hemopexin blocked this release, as well as chemotaxis of neutrophils in response to activated monocyte supernatants. These results suggest that hemopexin does not directly affect chemotaxis through protease activity, but may decrease heme-driven chemotaxis and secondary inflammation by attenuating the induction of chemoattractants from monocytes. This property could be beneficial in some settings to control potentially damaging inflammation induced by heme.
\end{abstract}

Online address: http://www.molmed.org

doi: $10.2119 / \mathrm{molmed} .2016 .00006$

\section{INTRODUCTION}

There has been increasing appreciation that cell-free plasma hemoglobin and secondarily released free heme may be toxic in critical care settings. Evidence for this concept comes from studies in which liberal transfusion is associated with equivalent or worse outcome than a more conservative transfusion strategy (1-4), studies suggesting that older stored blood is associated with higher morbidity than fresh blood (5-9), studies that older blood has higher levels of cell-free hemoglobin $(10,11)$, in vitro studies in which hemoglobin and heme potentiate release of cytokines from macrophages (12-14),

\section{${ }^{*} T L, J L$ and FH contributed equally.}

Address correspondence to Tian Lin, Department of Pediatrics, Infectious Disease Unit, Massachusetts General Hospital, Rm 5.234, $14913^{\text {th }}$ Street, Charlestown, MA 02129. Phone: 617-724-4995; Fax: 617-726-5411; E-mail: tlin7@mgh.harvard.edu. Submitted January 7, 2016; Accepted for publication January 7, 2016; Published Online (www.molmed.org) January 8, 2016.

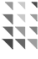

Feinstein Institute for Medical Research Northwell Health

animal models of endothelial toxicity (15), inflammation and sepsis $(15,16)$ and clinical studies in which elevated hemoglobin has been associated with worse outcomes in sepsis $(17,18)$.

Hemopexin $(\mathrm{Hx})$ is a heme-binding plasma glycoprotein that functions as a scavenger of free heme released from hemoglobin after rupture of red blood cells (RBCs). Hx binds heme with extraordinarily high affinity to form an oxidatively inert complex at the ratio of $1: 1\left(K_{d}<10^{-12} \mathrm{~mol} / \mathrm{L}\right)(19,20)$. Bound heme is neutralized and subsequently cleared into cells via the CD91 receptor. This binding affinity is so high that $\mathrm{Hx}$ "steals" heme from hemoglobin (21). Heme and hemoglobin are synergistic with toll-like receptor (TLR) agonists 
as well as endogenous mediators such as high-mobility group box protein 1 (HMGB1) to induce proinflammatory cytokines TNF and interleukin-6 (IL-6) from macrophages (12-14). Therefore, $\mathrm{Hx}$ has the potential to block the direct oxidative toxicity of free heme on tissues as well as the induction of proinflammatory cytokines induced synergistically by free heme and hemoglobin. Hx presumably serves to downregulate inflammation in clinical settings where extracellular heme may coexist with endogenous mediators or microbial components. Such settings include severe tissue infections, sepsis, trauma, burns, sickle cell crisis and acute respiratory distress syndrome (ARDS).

$\mathrm{Hx}$ has been proposed as a possible treatment for sepsis and sickle cell disease based upon studies finding that increased serum-free heme and decreased Hx were associated with worse outcomes (16-18) and that administration of $\mathrm{Hx}$ prevented the tissue damage and lethality in mouse models $(15,16)$. However, questions have been raised as to the safety of $\mathrm{Hx}$ infusion because of possible protease activity of $\mathrm{Hx}$ (22-24) and possible effects of $\mathrm{Hx}$ on neutrophil chemotaxis in a different study (25).

Since a variety of different Hx preparations purified from plasma using different techniques were used in prior studies, it has not been possible to determine if the different results stem from differences in the purity or activity of Hxs and in particular if the reported effects on chemotaxis and protease activities are intrinsic to the $\mathrm{Hx}$ molecule or are contaminations of the purified molecules with plasma proteases (26). To address this issue, we compared protease activities of Hxs from different sources including those used in many of the above studies, and studied their effects on neutrophil chemotaxis in vitro and neutrophil migration in vivo. We also created a recombinant $\mathrm{Fc}-\mathrm{Hx}$ fusion protein that allowed us to study properties of $\mathrm{Hx}$ in a molecule that was not purified from serum and that may be a candidate for clinical use.

\section{MATERIALS AND METHODS}

\section{Materials and Animals}

LPS from E. coli O55:B5 was purchased from List Biological Laboratories. Hemin chloride was purchased from Frontier Scientific Inc. Hemin solutions were made immediately before use in the dark as described previously (12). Chemoattractants N-formyl-L-methionylL-leucyl-L-phenylalanine (fMLF) and leukotriene B4 (LTB4) were purchased from Sigma-Aldrich and Cayman Chemical, respectively. Sterile human albumin aqueous solution [25\%] was purchased from Massachusetts General Hospital blood transfusion services, heat treated at $60^{\circ} \mathrm{C}$ for $10 \mathrm{~h}$ and saved in small aliquots at $-80^{\circ} \mathrm{C}$. C57BL $/ 6$ mice were obtained from Charles River Laboratories. The Institutional Animal Care and Use Committee at Massachusetts General Hospital approved the animal protocols used in this study.

Two purified human Hxs were used in the study: Hx purchased from Athens Research and Technology Inc. (hHx-A) and Hx produced at CSL Behring (hHx-B) (27). Rabbit Hx was prepared as described previously (28). A recombinant human $\mathrm{Hx}$ fusion protein was generated as a Fc fusion protein, Fc-hHx, which consists of the tripeptide alanine-lysine-threonine (AKT) followed by the hinge $\mathrm{CH} 2$ and $\mathrm{CH} 3$ domains of human IgG1 Fc (residues E216-G446) with two point mutations (C220S and N297D) and a single alanine linker fused with the full length sequence of the mature Hx polypeptide. This recombinant Fc-hHx was analyzed using SDS-PAGE and was shown to be a 150-kDa protein with $>95 \%$ purity on Coomassie Fluor Orange (Invitrogen) staining and Western Blot analysis using Cy3 labeled, goat anti-human Fc antibody (Jackson ImmunoResearch).

\section{Amidolytic Assay}

Amidolytic Assays on Hxs were performed as described previously with modifications (25). Two serine protease chromogenic substrates were used to perform amidolytic assay: H-D-IIe-ProArg-pNA.2HCl (S2288) that is sensitive to a broad spectrum of serine protease, and H-D-Phe-Pip-Arg-pNa-2HCl (S2238) that is sensitive to thrombin. Both substrates were purchased from Diapharma Group Inc. The assay was performed according to the instructions of the manufacturer with minor modifications.

\section{Macrophage Culture and Cytokine Assays}

Bone marrow-derived macrophages (BMDMs) were prepared from mice as we described previously (12). BMDMs were washed three times in serum-free medium, followed by incubation overnight with LPS with or without hemin or hemoglobin in the presence or absence of Fc-hHx or Fc or hHx-B in the culture as noted. Concentrations of TNF in the supernatants were quantitated by enzyme-linked immunosorbent assay (ELISA) (R\&D Systems) according to the manufacturer's instructions.

\section{Heme Binding Assay}

Hemin chloride, the synthesized form of free heme was prepared as described previously (29). Protein samples $(10 \mu \mathrm{mol} / \mathrm{L})$ or PBS were mixed with hemin at different concentrations (3 and $10 \mu \mathrm{mol} / \mathrm{L}$ ) and incubated at room temperature for $30 \mathrm{~min}$. The spectra of hemin-protein were recorded by Nanodrop ND1000. Hemin-hemopexin complexes form an absorption peak at 413 nanometers ( $\mathrm{nm}$ ) (30). The absorbance at $413 \mathrm{~nm}$ was measured and compared among samples with different molar ratios of protein and hemin (3:1 and1:1).

\section{Isolation of Human Peripheral Neutrophils and Monocytes}

Fresh human neutrophils and monocytes were isolated as described previously with minor modifications $(31,32)$. Human studies were approved by the Institutional Review Board at the Massachusetts General Hospital (assurance \# FWA00003136). Briefly, 
fresh heparinized human blood was carefully layered on the top of two layers of Ficoll gradient (Histopaque 1077 and 1119, Sigma) followed by centrifugation at $700 \times g$ with brake off for $30 \mathrm{~min}$ at room temperature. The cells in the layer on the top of Histopaque 1077 were washed in HBSS without calcium (Life Technologies) and plated in 48-well tissue culture plate with $250 \mu \mathrm{L} /$ well $\left(4 \times 10^{6} / \mathrm{mL}\right)$ in RPMI with L-glutamine and $1 \%$ streptomycin/penicillin, then incubated at $37^{\circ} \mathrm{C}$ in humidified air with $5 \% \mathrm{CO}_{2}$ for $1 \mathrm{~h}$, followed by five washes in RPMI to remove nonadherent cells. The adherent cells were used as monocytes for desired experiments. Neutrophils were recovered at the interface of Histopaque 1077 and 1119 and washed with HBSS without calcium (Life Technologies). Contaminating erythrocytes were removed by Red Blood Cell Lysis Buffer (Sigma). Neutrophils were $>95 \%$ pure as determined by morphologic analysis of May-Grumwald-Giemsa (Merck) stained cytopreparations. Neutrophils were suspended in RPMI 1640 containing $2 \%$ fetal bovine serum.

\section{Culture of Human Peripheral Blood Monocytes and Measurement of LTB4 by EIA}

Human monocytes were incubated with LPS $(10 \mu \mathrm{g} / \mathrm{mL})$ in the presence or absence of hemin $(1 \mu \mathrm{mol} / \mathrm{L}$ or $10 \mu \mathrm{mol} / \mathrm{L})$ prepared as described previously (12) for 1, 3, $5 \mathrm{~h}$. In some experiments, $\mathrm{hHx}-\mathrm{B}$ was added to the culture. The supernatants were saved at $-80^{\circ} \mathrm{C}$ for later LTB4 measurement or used in the chemotaxis assay of human neutrophils. LTB4 production was measured by Enzyme Immunoassay Kit according to the manufacturer's instructions (Cayman Chemical).

\section{Human Neutrophil Chemotaxis Migration Assay}

Chemotaxis migration was performed in a 96-well cell migration system with 5 - $\mu \mathrm{L}$ pore size, $30 \mu \mathrm{L}$-plate well volume and $8 \mathrm{~mm}^{2}$ filter area (Neuro Probe Inc.). Human neutrophils were preincubated with different Hxs at $100 \mu \mathrm{g} / \mathrm{mL}$ $(1.67 \mu \mathrm{mol} / \mathrm{L})$ or different controls (FC or RPMI 1640 containing 2\% FBS) at $37^{\circ} \mathrm{C}$ in humidified air with $5 \% \mathrm{CO}_{2}$ for $1 \mathrm{~h}$, and then transferred to each site of the filter top $\left(1-2.5 \times 10^{5}\right.$ cells $/ 30 \mu \mathrm{L} /$ well). The lower microplate wells were filled with different concentrations of fMLF or LTB4 as desired. After incubation at $37^{\circ} \mathrm{C}$ in humidified air with $5 \% \mathrm{CO}_{2}$ for $30 \mathrm{~min}$ to $1.5 \mathrm{~h}$, the filter was removed. Neutrophils migrated to the bottom microplate wells were stained with $0.4 \%$ trypan blue (Life Technologies) and counted.

\section{Neutrophil Migration in Mouse Peritoneal Cavity}

Neutrophil migration into the peritoneal cavity of mice was induced by i.p. injection of thioglycollate and was measured in mice with administration of Hx as described by Spiller et al. (25). The Institutional Animal Care and Use Committee at Massachusetts General Hospital approved the animal protocols used in this study. Thirty minutes after intravenous Hx or albumin ( $2 \mathrm{mg} /$ mouse) administration, neutrophil migration was induced by i.p. injection of $500 \mu \mathrm{l} \%$ thioglycolate (Sigma). At $2 \mathrm{~h}$ and $4 \mathrm{~h}$ after thioglycolate administration, the peritoneal cells were washed with $5 \mathrm{~mL}$ of PBS twice and harvested after gentle massage. The cells were centrifuged at $500 \times g$ for $5 \mathrm{~min}$ at $4^{\circ} \mathrm{C}$, stained with $0.4 \%$ trypan blue and the total cell numbers were determined by counting under light microscope using hemocytometer. The cells were applied to flow cytometry analysis or MPO assay.

\section{Flow Cytometry Analysis}

Cells from mice peritoneal cavity were washed in $\mathrm{Ca}^{2+}$ and $\mathrm{Mg}^{2+}$-free HBSS containing 5\% heat-inactivated fetal bovine serum and $5 \mathrm{mmol} / \mathrm{L}$ EDTA. For flow cytometry immunophenotyping, $3 \times 10^{6}$ cells per tube were incubated in $0.5 \mu \mathrm{g}$ Fc Block (BD Biosciences) for $10 \mathrm{~min}$ at room temperature, and then incubated with
PE-conjugated anti-Gr-1 (RB6-8C5) $\mathrm{mAbs}$ (BD Biosciences) in the dark for $30 \mathrm{~min}$ at $4^{\circ} \mathrm{C}$. Cells were then washed twice with staining buffer followed by fixation in $1 \%$ paraformaldehyde (Thermo Scientific). The cells were analyzed on a FACSCalibur flow cytometer (BD Biosciences) equipped with FACSDIV software to determine the percentage of neutrophils over total cells migrated to the peritoneal cavity.

\section{Myeloperoxidase (MPO) Assay}

The MPO activity was measured in neutrophils recruited to the peritoneal cavity of mice. Cells harvested from the peritoneal cavity after thioglycolate administration were resuspended in $0.5 \%$ HTAB (hexadecyl-trimethyl-ammonium bromide) in $50 \mathrm{mmol} / \mathrm{L}$ potassium and sodium phosphate, $\mathrm{pH} 6.0$ and sonicated on ice by microtip at $3 \times 10 \mathrm{~s}$ bursts with $90 \%$ duty cycle and power setting in a Branson 450 sonicator (Branson Ultrasonics Corp.). The cells were then put in an ethanol/dry ice bath alternating with warm room at $37^{\circ} \mathrm{C}$. After three sonicatefreeze-thaw cycles, the samples were finally sonicated for $10 \mathrm{~s}$ on ice, followed by centrifugation at $12,000 \times g$ for 30 $\min$ at $4^{\circ} \mathrm{C}$. The supernatants were saved at $-80^{\circ} \mathrm{C}$ for MPO assay. The enzyme activity was determined spectrophotometrically as the MPO-catalyzed change in absorbance in the redox reaction of $\mathrm{H}_{2} \mathrm{O}_{2}$ (450 nm with a reference filter 620 $\mathrm{nm}, 25^{\circ} \mathrm{C}$ ), as described previously (22). MPO concentrations (mUnits/peritoneal cavity) were determined using $\mathrm{MPO}$ standards (Sigma).

\section{Statistical Analysis}

Except where indicated, representative data from at least three experiments are presented in the Figures. Data are expressed as means, and error bars represent SE. The data were analyzed by GraphPad Prism 6 (GraphPad Software). Unpaired $t$ tests were performed to compare treated samples with controls. Values of $p<0.05$ (two-tailed) were considered statistically significant. 


\section{RESULTS}

\section{Serine Protease Activity of Purified Hxs}

To study protease activity of Hxs, amidolytic assays were performed by using two serine protease chromogenic substrates: S2238 (for thrombin) and S2288 (for general serine protease activity) evaluated over a 48-h time span. Hxs were studied at concentrations of $6.7 \mu \mathrm{mol} / \mathrm{L}$ (400 $\mu \mathrm{g} / \mathrm{mL})$, which is similar to what might be achieved in clinical studies. Protease activity varied substantially between the preparations (Figure 1).

\section{Purified Hx Does Not Inhibit the Chemotaxis of Neutrophils Induced by fMLF and LTB4}

The effect of Hx on chemotaxis was investigated in vitro using two chemoattractants, fMLF and LTB4, in the transmigration assay. Freshly isolated human peripheral neutrophils were preincubated with either RPMI with $2 \%$ FBS as a control or the purified Hxs at the concentration of $100 \mu \mathrm{g} / \mathrm{mL}$ $(1.67 \mu \mathrm{mol} / \mathrm{L})$. Both fMLF and LTB4 induced a marked dose-dependent increase in human neutrophil migration. However, there was no significant effect of any of the Hxs on chemotaxis (Figures 2A, B).

\section{Recombinant Fc-hHx Binds Heme and Inhibits Heme-LPS Synergy on Monocytes}

A recombinant $\mathrm{Hx}$ molecule might have some advantages over Hx purified from plasma for study of Hx properties, as well as for possible eventual clinical use. Initial attempts at generating hHx were complicated by poor expression that limited an adequate yield. This problem was solved by the creation of Fc-hHx, consisting of a fusion protein of the Fc region of IgG1 and $\mathrm{Hx}$ that was expressed in mammalian cells. Details of the production of the Fc-hHx are included in Methods. This material is endotoxin-free as tested by LAL and has high purity according to SDS-PAGE (Figure 3A) and Western blot results (Figure 3B).
We found that the recombinant Fc$\mathrm{hHx}$ bound heme as well as purified hHx (Figure 3C), and had comparable activity to purified hHx to inhibit the induction of TNF from macrophages synergistically induced by heme and LPS (Figure 4).

\section{Recombinant Fc-Hhx has Minimal Protease Activity and Does Not Inhibit Chemotaxis of Neutrophils}

A potential problem in studying purified hHx to evaluate if there may be intrinsic protease activity is that these preparations have some risk of contamination by other serum proteins $(22,24)$. Study of a recombinant version should minimize this issue. There was extremely low protease activity in the Fc-hHx using the same two protease activity assays using substrate S2288 and S2238 (Figures 5A, B). However, close examination of the curves (inserts in Figures 5A, B) suggested that there was an immediate very small increase in absorbance at $405 \mathrm{~nm}$ wavelength
A

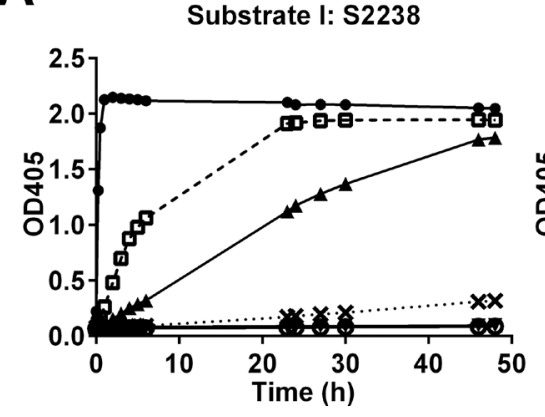

B

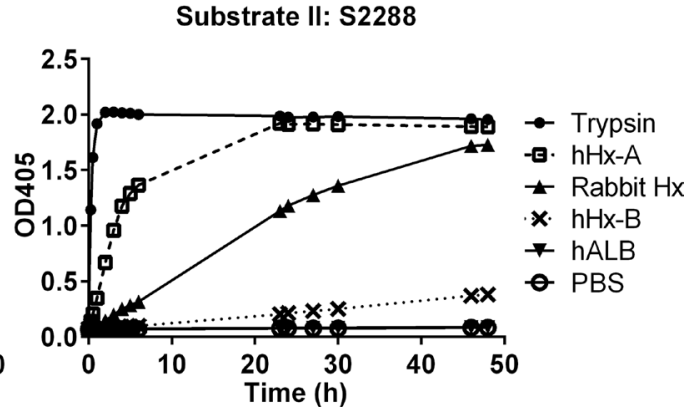

Figure 1. Protease activity of purified Hxs. Enzymatic activity of different purified Hxs (two human and one rabbit) was tested at the same molar concentration ( $6.7 \mu \mathrm{mol} / \mathrm{L}$ ) using chromogenic substrate I: S2238 and II: S2288 that are sensitive to thrombin and serine proteases respectively. Trypsin $(0.4 \mu \mathrm{mol} / \mathrm{L})$ was used as positive control. The substrates were mixed with different $\mathrm{Hxs}$ or controls and incubated at $37^{\circ} \mathrm{C}$ for different times. The absorbance at $405 \mathrm{~nm}$ was measured by spectrophotometry. The results represent mean $\pm \mathrm{SE}$ and are representative of three independent experiments.

A

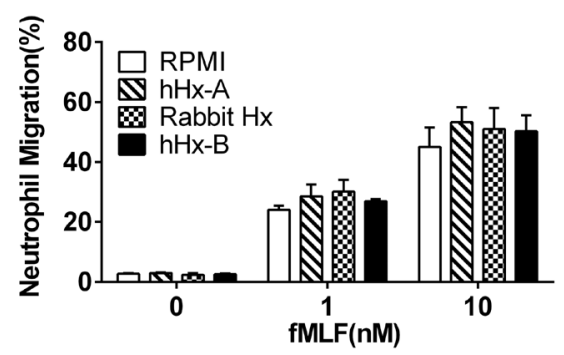

B

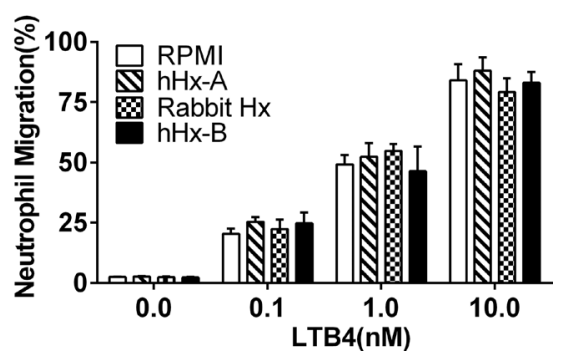

Figure 2. Purified Hxs do not inhibit the chemotaxis of neutrophils induced by fMLF and LTB4. Human neutrophils were preincubated with different purified $\mathrm{Hxs}$ ( $\mathrm{hHx}-\mathrm{A}$, rabbit $\mathrm{Hx}$ and $\mathrm{hHx}-\mathrm{B}$ ) or control (RPMl 1640 containing $2 \%$ FBS) for $1 \mathrm{~h}$. Then $1-2.5 \times 10^{5}$ cells $/ 30 \mu \mathrm{l}$ cells were applied to the top of the transwell with the lower microplate filled with $\mathrm{fMLF}$ ( $1 \mathrm{nmol} / \mathrm{L}$ and $10 \mathrm{nmol} / \mathrm{L}$ ) (A) or LTB4 $(0.1 \mathrm{nmol} / \mathrm{L}, 1 \mathrm{nmol} / \mathrm{L}$ and $10 \mathrm{nmol} / \mathrm{L}$ ) (B) and incubated for $1 \mathrm{~h}$. Neutrophils migrated to the bottom microplate wells were stained with $0.4 \%$ trypan blue and live cells were counted. The percentage of cells migrated over the total cells uploaded to the top of the transwell was calculated. The results represent the mean $\pm \mathrm{SE}$ and are representative of three independent experiments. 

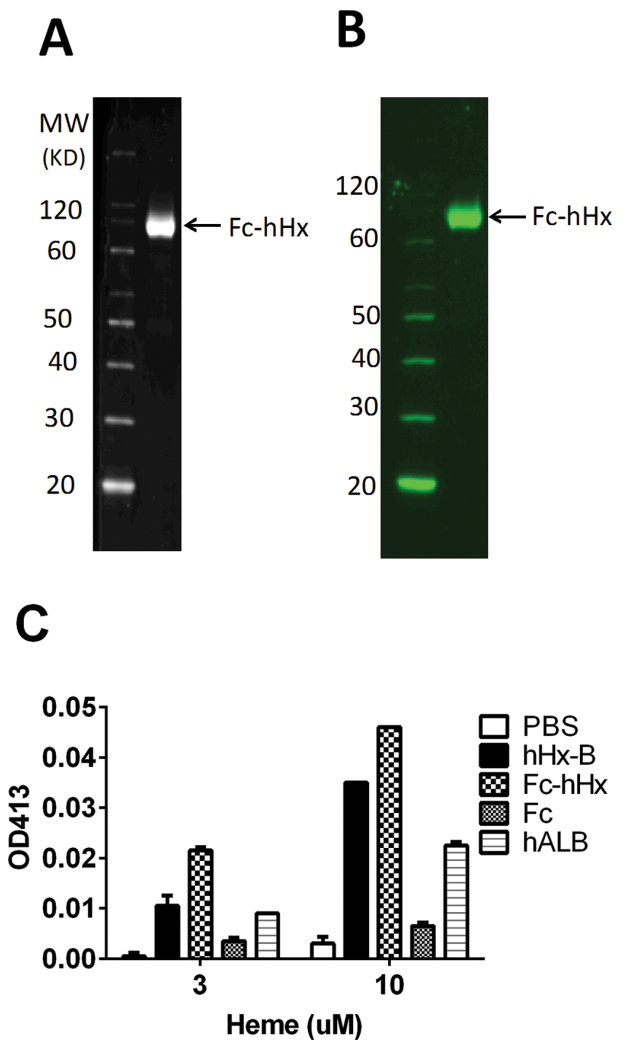

Figure 3. Recombinant Fc-hHx has the same heme-binding activity as purified $\mathrm{Hx}$. Recombinant Fc-hHx prepared as described in Materials and Methods was applied to SDS-PAGE with Coomassie Fluor Orange (Life Technologies) staining (A) and developed as a Western blot with Cy3-labeled goat anti-human Fc antibody (Jackson ImmunoResearch) (B). Fc-hHx is shown in the reduced gels as a high-purity 75-kDa monomer (see arrow). In nonreduced gels, the Fc-hHx is a $150-k D a$ dimer (not shown). (C) Heme binding assay was done with $10 \mu \mathrm{mol} / \mathrm{L}$ of recombinant Fc-hHx; purified $\mathrm{hHx}-\mathrm{B}$; Fc fragment of IgGl; human albumin (hALB). All protein samples or PBS were mixed with free heme at different concentrations ( 3 and $10 \mu \mathrm{m}$ ) at room temperature for $30 \mathrm{~min}$. The absorbance at $413 \mathrm{~nm}$ was measured by Nanodrop ND1000. The results represent the mean \pm SE.

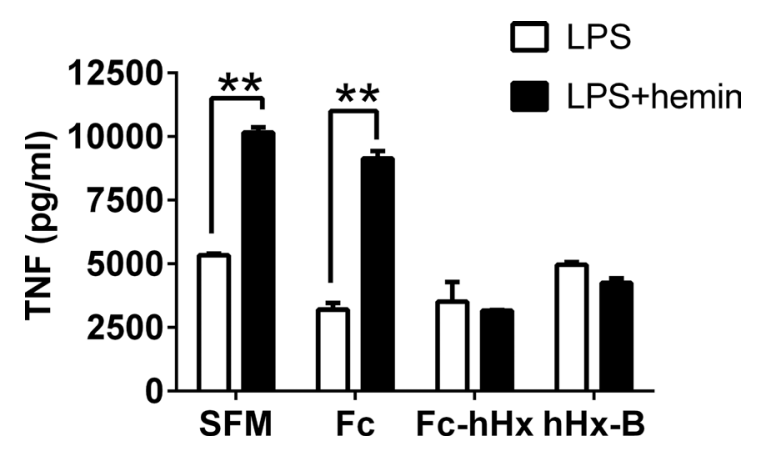

Figure 4. Recombinant Fc-hHx inhibits TNF production synergistically induced by LPS and hemin. Mouse BMDMs were incubated with LPS ( $2 \mathrm{ng} / \mathrm{mL})$ only or with hemin $(1 \mu \mathrm{mol} / \mathrm{L})$ in serum-free medium (SFM) for $9 \mathrm{~h}$ in the absence or presence of $1.67 \mu \mathrm{mol} / \mathrm{L}$ of one of the following proteins: Fc fragment of IgG 1; Fc-hHx; hHx-B. TNF levels were measured in the supernatants of the culture. The results represent mean \pm SE and are representative of four independent experiments. ${ }^{* *} P<0.01$, compared between cells treated with and without hemin. with Fc-hHx after addition of the substrates that was not seen with the Fc control, and that the slope of the curve was slightly positive. Fc-hHx had no effect on human neutrophil chemotaxis (Figures 5C, D).

\section{Hx Does Not Suppress Neutrophil Migration in Mice}

An earlier article reported that purified Hx suppressed the neutrophil migration into the peritoneal cavity in a mouse model in which chemotaxis was induced by thioglycolate or with sepsis induced by cecal ligation puncture (25). We tested the effect of $\mathrm{Hx}$ on neutrophil migration in mice in a similar way. Mice were injected i.v. with Hx or control human albumin at the dose of $2 \mathrm{mg} /$ mouse $30 \mathrm{~min}$ before induction of chemotaxis by i.p. injection of thioglycolate. There was no significant difference in neutrophil numbers (Figure 6A) or myeloperoxidase activity (Figure 6B) in the peritoneal cavity between the mice treated with Hx and albumin.

\section{Hx Blocks Synergistic Production of LTB4 Induced by Hemin and LPS}

Heme and LPS synergistically induce the production of TNF and IL- 6 from macrophages (12). Accordingly, we studied the effect of heme and LPS on the production of chemoattractants from human monocytes. LTB4 levels in supernatants of monocytes stimulated with LPS increased with time and peaked at $3 \mathrm{~h}$, similar to prior studies with human alveolar macrophages (33). Heme alone induced a slight increase in LTB4 (34). However, the combination of LPS and heme $(1 \mu \mathrm{mol} / \mathrm{L})$ led to significantly higher concentrations of LTB4 in the culture medium (Figure 7A). Similar results were found with the combination of LPS and hemin at $10 \mu \mathrm{mol} / \mathrm{L}$ (data not shown). The addition of $\mathrm{hHx}$ blocked the synergistic increase in LTB4 production to the level of chemotaxis induced in the absence of heme (Figure 7B). Similarly, there was a synergistic increase in chemotaxis of 

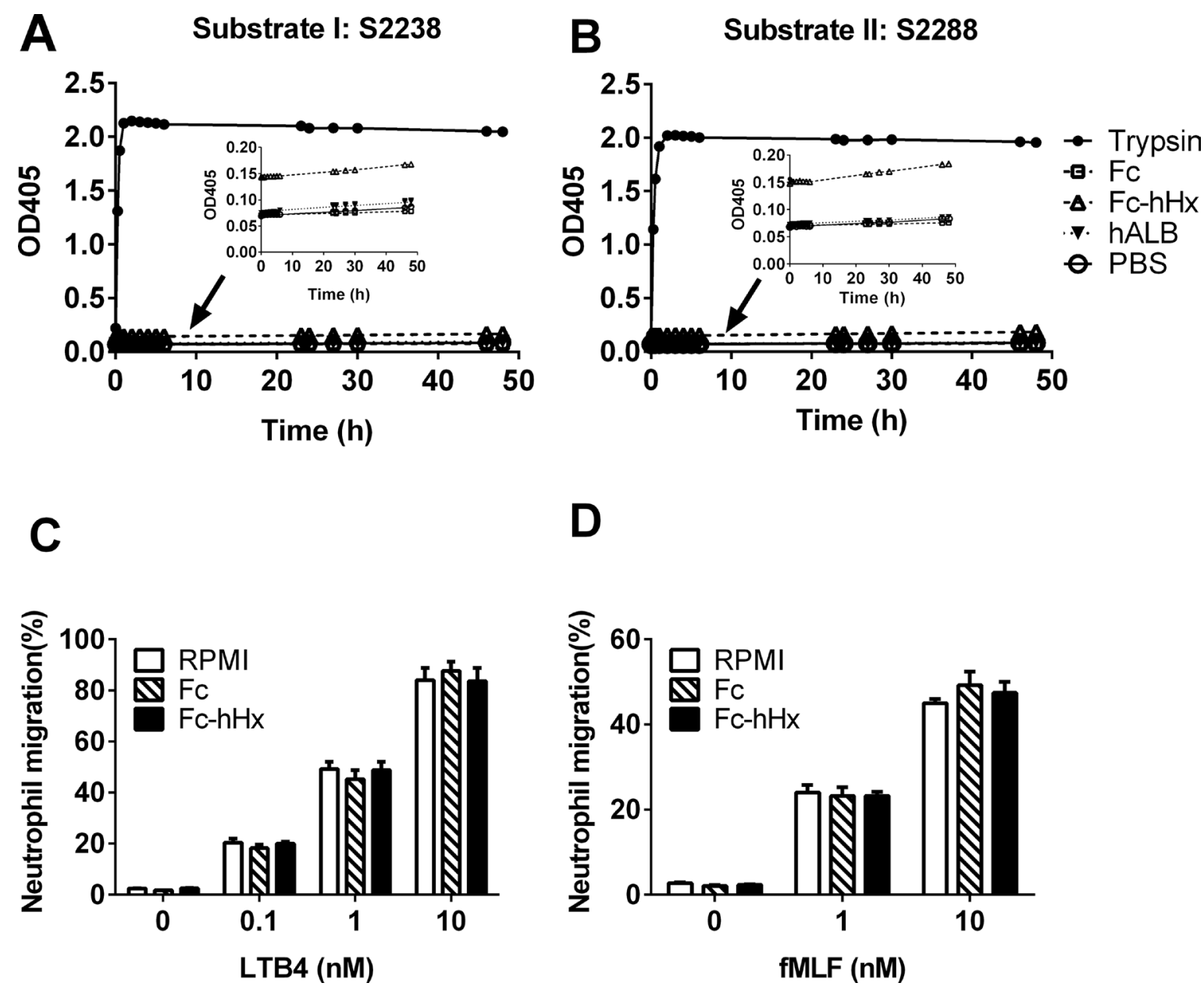

Figure 5. Recombinant Fc-hHx has minimal protease activity and no inhibition on the chemotaxis of human neutrophils induced by fMLF and LTB4. Enzymatic activity of recombinant Fc-human $\mathrm{Hx}(\mathrm{Fc}-\mathrm{hHx}$ ) was tested by using chromogenic substrates substrate I: S2238 (A) and II: S2288 (B). Fc-hHx and the tag Fc at the concentration of $6.7 \mu \mathrm{mol} / \mathrm{L}$, positive control trypsin $(0.4 \mu \mathrm{mol} / \mathrm{L})$, negative control human albumin (hALB) and PBS were mixed with the substrate at desired concentrations and incubated at $37^{\circ} \mathrm{C}$ for different times. The absorbance at $405 \mathrm{~nm}$ was measured by spectrophotometry. The small inserts are the sections with small scale and without trypsin control to show the very low increase of the absorbance by Fc-hHx. (C) and (D): Fc or Fc-hHx at $100 \mu \mathrm{g} / \mathrm{mL}(1.67 \mu \mathrm{mol} / \mathrm{L}) \mathrm{were}$ also used to pretreat human neutrophils with control (RPMl 1640 containing $2 \%$ FBS) for $1 \mathrm{~h}$. Then $1-2.5 \times 10^{5}$ cells $/ 30 \mu \mathrm{L}$ cells were applied to the top of the transwell with the lower microplate filled with LTB4 $(0.1 \mathrm{nmol} / \mathrm{L}, 1 \mathrm{nmol} / \mathrm{L}$ and $10 \mathrm{nmol} / \mathrm{L})(\mathrm{C}) \mathrm{or} \mathrm{fMLF}(1 \mathrm{nmol} / \mathrm{L}$ and $10 \mathrm{nmol} / \mathrm{L}$ ) (D) and incubated for $1 \mathrm{~h}$. Neutrophils migrated to the bottom microplate wells were stained with $0.4 \%$ trypan blue and live cells were counted. The percentage of cells migrated over the total cells uploaded to the top of the transwell was calculated. The results represent the mean \pm SE and are representative of three independent experiments.

neutrophils exposed to supernatants of monocyte culture with hemin and LPS, and the hemin-induced component of this increase was eliminated in the presence of Hx (Figure 7C). Together, these findings support a mechanism by which Hx specifically decreases heme-induced chemotaxis by blocking the synergistic induction of chemoattractants (Figure 8).

\section{DISCUSSION}

$\mathrm{Hx}$ is a plasma glycoprotein with extraordinary heme binding affinity that has been proposed as a candidate drug to decrease heme-induced inflammation in numerous settings $(12,14)$. The major findings of the current study are that the purified Hxs obtained from different sources that are similar to those used in prior studies had large differences in protease activities. A recombinant $\mathrm{Hx}$ fusion protein consisting of Hx coupled to the Fc moiety of IgG bound heme well and had detectable but minimal protease activity. None of the Hxs studied had a significant effect on chemotaxis of neutrophils in vitro or in vivo. We, in addition, found that heme synergizes with LPS to induce the production of chemoattractant LTB4 from monocytes and that 


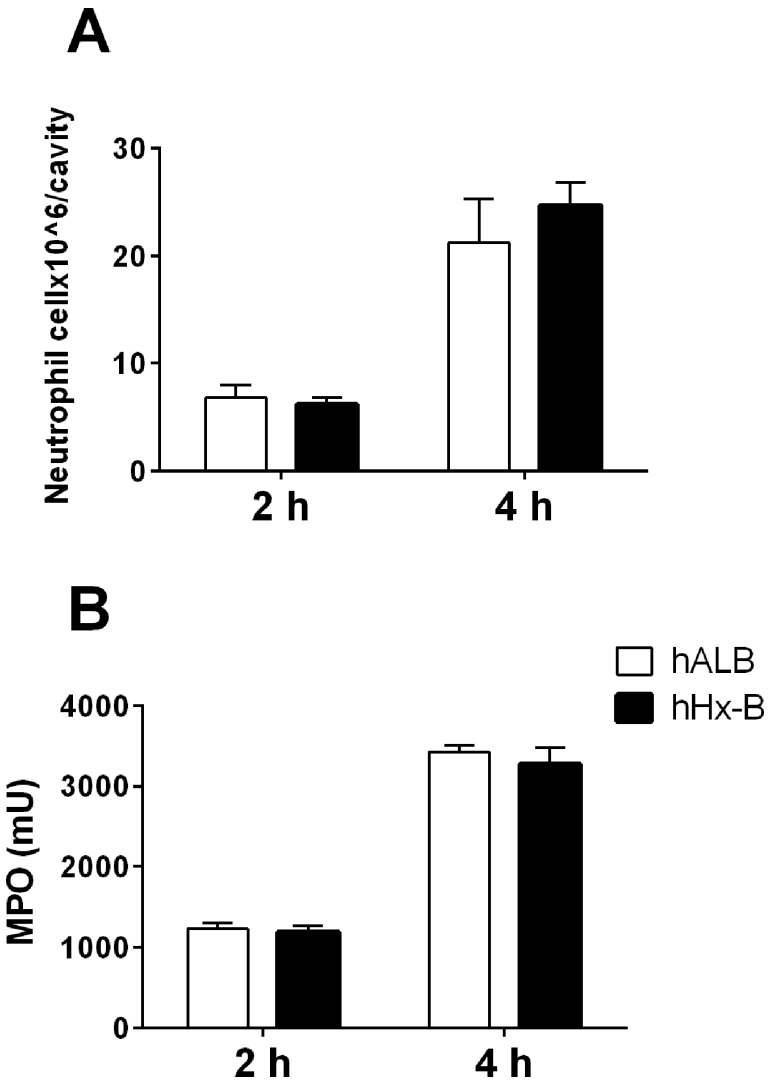

Figure 6. Hx does not suppress neutrophil migration in mice. Thirty minutes after intravenous injection of human $\mathrm{Hx}(\mathrm{hHx}-\mathrm{B})$ or albumin (hALB) $(2 \mathrm{mg} / \mathrm{mouse})$, neutrophil migration was induced by i.p. injection of $500 \mu$ of $4 \%$ thioglycolate. At $2 \mathrm{~h}$ or $4 \mathrm{~h}$ post injection, peritoneal cells were flushed out by PBS and counted. (A) The peritoneal cells were incubated with PE- conjugated anti-Gr-1 and analyzed via FACS to determine the percentage of neutrophils over the total cells. Total neutrophils were calculated. (B) The MPO activity was measured in the total peritoneal cells. The results are representative of five independent experiments and presented as the mean $\pm \mathrm{SE}$.

Hx blocks this synergy, resulting in the decreased chemotaxis induced by this synergy.

Cell-free hemoglobin is released from damaged RBCs into tissues or plasma in numerous clinical settings with or without infection, as well as after transfusion. Patients with ARDS have evidence of increased RBCs and hemoglobin in tissues on histological sections (35). Studies in mice also indicate that there is hemorrhage and free hemoglobin in the intraalveolar space following intratracheal injection of LPS (36). In studies of patients with sepsis, elevated free plasma hemoglobin correlated with mortality $(17,18)$, and a common feature is endothelial cell dysfunction caused by cell-free hemoglobin (37) that may cause secondary organ failure in the lung. The reported sensitivity of endothelium to heme and the frequent physical presence of blood in alveoli may make the lung potentially and uniquely sensitive to heme-driven pathophysiology (38). Heme and hemoglobin synergize with TLR agonists, bacteria and HMGB1, inducing high levels of proinflammatory cytokines TNF and IL-6 from macrophages (12-14), and Hx blocks this synergy (12-14), either through direct sequestering of free heme, or possibly indirectly through activation of heme oxygenase-1 (HO-1), inducing the production of antiinflammatory $\mathrm{CO}$ and biliverdin $(39,40)$. Larsen et al. showed increased serum-free heme in mouse sepsis model of cecal ligation and puncture, and administration of rabbit Hx prevented the tissue damage and lethality (16). Therefore, replenishing Hx could be a potential therapeutic strategy to control heme-driven inflammation in critical care patients.

Some prior studies have suggested that Hx might have intrinsic protease activity. Bakker et al. identified a plasma factor $100 \mathrm{KF}$ that is closely related to $\mathrm{Hx}$ and has protease activity that induced transient protein leakage after incubation with rat kidney ex vivo and perfusion of kidney $(22,23)$. In a different study, protease activity was reported in recombinant $\mathrm{Hx}$ expressed in Pichia pastoris using an amidolytic assay, although there was no expressed control protein reported (24). Spiller et al. reported that a preparation of purified human Hx suppressed the chemotaxis of neutrophils and increased mortality in a mouse sepsis model with cecal ligation, and that these effects were prevented by protease inhibitors (25). These studies raised the question of the safety of infusing $\mathrm{Hx}$ into humans. To address this issue, and to help interpret the results of some of the prior and conflicting animal studies in the literature, we tested the protease activity and the effect on chemotaxis of Hx from four different sources that include preparations of rabbit and human Hx similar to those used in prior animal studies (rabbit $\mathrm{Hx}$ and two preparations of human $\mathrm{Hx}$ ) as well as a novel recombinant human $\mathrm{Hx}$ fused with $\mathrm{F}_{\mathrm{C}}$ that we produced and expressed in $\mathrm{CHO}$ cells. We found that protease activity varied considerably in the different Hx preparations (Figure 1), but that none of the Hx preparations altered neutrophil migration (Figures 2,5C,5D). In further 


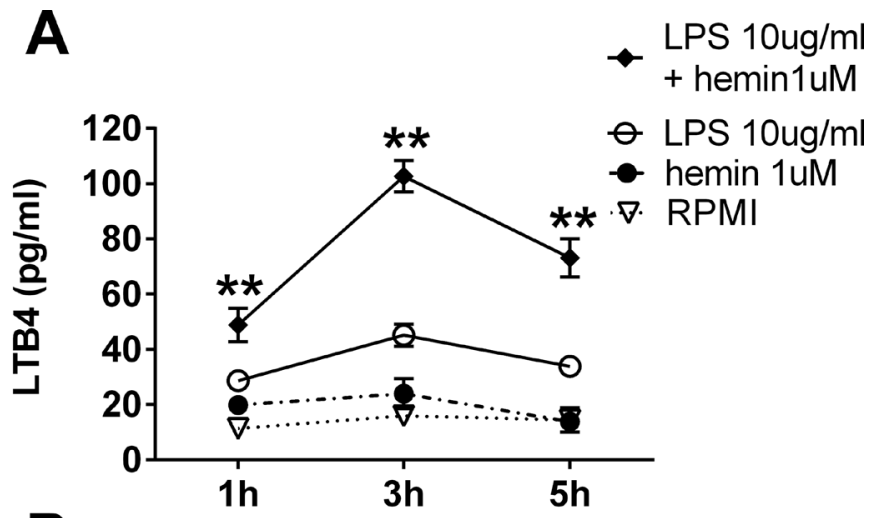

B
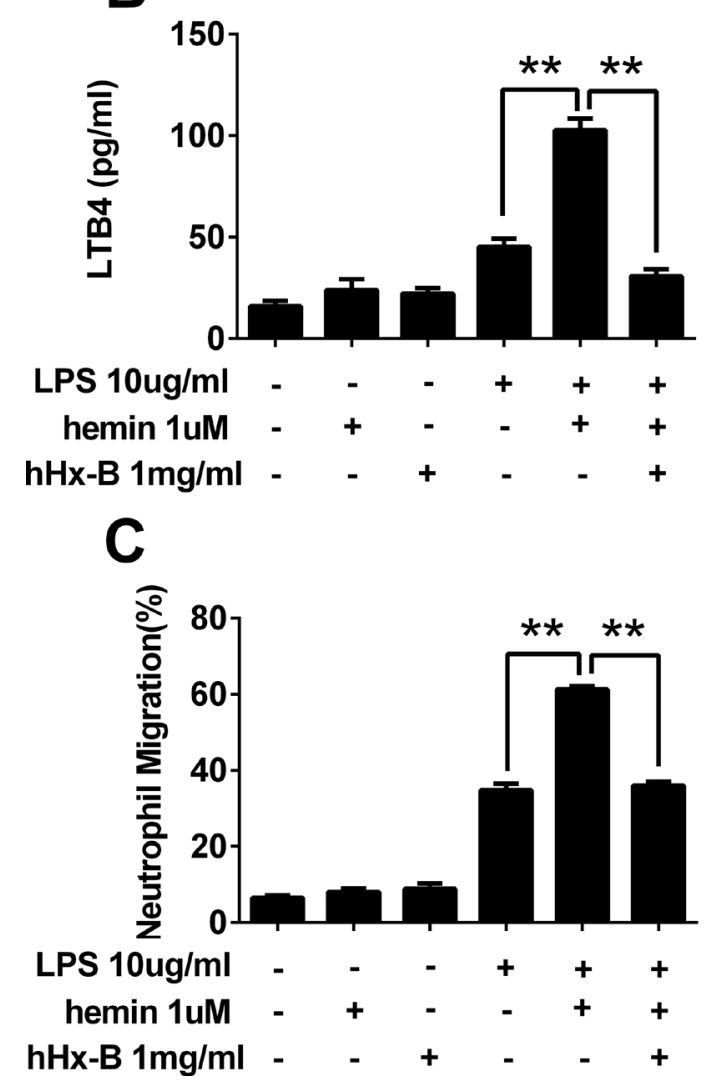

Figure 7. LPS and hemin synergistically induce the production of LTB4 from human monocytes and Hx blocks this synergy by decreasing LTB4 levels and suppresses chemotaxis of neutrophils. (A) Human monocytes were incubated with LPS $(10 \mu \mathrm{g} / \mathrm{mL})$ in the presence or absence of hemin $(1 \mu \mathrm{mol} / \mathrm{L})$ for 1,3 and $5 \mathrm{~h}$. LTB4 concentrations in the supernatants were measured and compared between the cultures with LPS only and with LPS and hemin. (B) Human monocytes were incubated with LPS $(10 \mu \mathrm{g} / \mathrm{mL})$ and with or without hemin $(1 \mu \mathrm{mol} / \mathrm{L})$ in the absence or presence of $\mathrm{hHx}-\mathrm{B}(1 \mathrm{mg} / \mathrm{mL})$ for $3 \mathrm{~h}$. LTB4 concentrations were measured and compared between the cultures with and without $H x$. (C) The supernatants collected from human monocyte cultures at $3 \mathrm{~h}$ were loaded to the bottom microplate well of the transmigration system in chemotaxis assay. The percentage of neutrophils migrated to the bottom wells over the total cells loaded to the top of the transwells were calculated and compared between the cultures with and without $\mathrm{hHx}-\mathrm{B}$. The results are representative of three independent experiments and presented as the mean $\pm \mathrm{SE} .{ }^{* *} P<0.01$ LPS with hemin versus LPS only. studies, hHx-B did not inhibit neutrophil migration to the peritoneal cavity induced by thioglycolate (Figure 6).

The generation of a recombinant Fc-hHx preparation allowed us to study a preparation of $\mathrm{Hx}$ that should not have contaminating proteases remaining from plasma purification. We compared this reagent to a recombinant Fc control and to human albumin. Although barely detectable, we consistently found that there was trace protease activity using both substrates studied compared with the two control preparations (Figures 5A, B). This finding may suggest that there is an isoform of $\mathrm{Hx}$ that contains intrinsic protease activity, albeit at trace levels. However, we cannot absolutely exclude the possibility that this trace activity resulted from a purification contaminant from the host cells present in this preparation but not the controls.

The availability of a recombinant version of $\mathrm{Hx}$ in the form of an $\mathrm{Fc}-\mathrm{hHx}$ fusion protein raises the question of whether it might be a suitable as a potential therapeutic candidate to replenish $\mathrm{Hx}$ in situations of heme excess. We found that the fusion protein had similar heme-binding activity and cytokine-blocking activity as purified human Hx (Figures 3C, 4), and did not inhibit neutrophil migration (Figures 5C, D). Further studies are needed to study and compare Fc-hHx and purified $\mathrm{hHx}$ in animal models.

The earlier report that Hx suppressed neutrophil migration led to the hypothesis that the suppression may be due to protease activity in the preparation that directly acted on neutrophils to decrease chemotaxis (25). An alternative possibility would be that hemopexin bound cell-free heme that induced or primed macrophages to produce chemotaxins such as leukotriene B4 (LTB4). LTB4 has been described as the major chemotaxin induced by heme (34). In our studies, none of the $\mathrm{Hx}$ preparations, regardless of protease activity, directly suppressed neutrophil migration. To evaluate the possibility that Hx might suppress chemotaxis by binding heme, we studied 


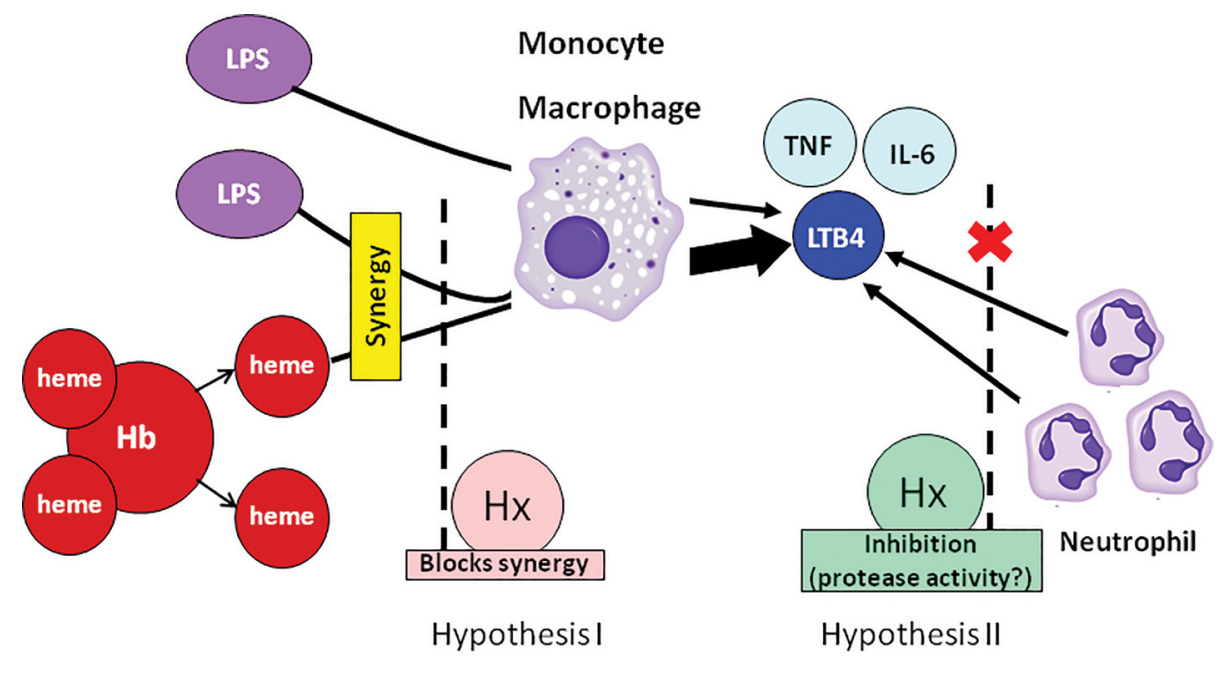

Figure 8. The role of $\mathrm{Hx}$ in neutrophil chemotaxis. The pathogenesis of severe infectious diseases is associated with hemolysis and secondary release of cell-free heme. LPS and heme synergize to induce the production of TNF, IL-6 and LTB4 from monocytes/ macrophages. LTB4 induces neutrophil migration. Two hypotheses might explain the effect of Hx to suppress chemotaxis of neutrophils: hypothesis I, Hx binds heme and therefore blocks synergistic production of LTB4 and other chemotaxins, secondarily decreasing the signal for the chemotaxis of neutrophils; hypothesis II, Hx itself has protease activity and direct inhibitory effect on the neutrophil migration. The results from this paper support hypothesis I.

the synergy of hemin and LPS to induce LTB4 from isolated macrophages, and the ability of a purified Hx with very low protease activity to suppress this induction. We found that LPS and hemin are synergistic for LTB4 production, that $\mathrm{Hx}$ blocks this production and that Hx also blocks the chemotaxis induced by LTB4 containing cell supernatants (Figure 7). These findings support the concept that Hx decreases heme-induced secondary inflammation by binding heme and blocking its activity (hypothesis I in Figure 8).

\section{CONCLUSION}

The finding that LPS synergizes with hemin to induce LTB4 may explain in part why intra-or extravascular hemoly sis leads to only mild inflammation, whereas small amounts of heme in the presence of a secondary signal such as LPS, may greatly amplify it. This finding might be particularly important in infected lungs given their sensitivity to LTB4. Our data add to the body of evidence that extracellular heme is a cause of tissue inflammation, and is consistent with a strategy of infusion of Hx to replenish depleted native local or systemic $\mathrm{Hx}$ in settings of excess and toxic heme. Our failure to detect direct neutrophil suppression by multiple different $\mathrm{Hx}$ preparations, irrespective of different protease activities is reassuring regarding the possible development of a safe hemopexin product for clinical testing.

\section{ACKNOWLEDGMENTS}

This work was funded by the National Institutes of Health (AI059010), the Shriners Hospital for Crippled Children (87200, 84298), DARPA (W911NF-13-1-0070, W911NF-10-1-0271, N66001-11-14180), and Wang Kuancheng Fund from Ruijin Hospital, Shanghai Jiaotong University School of Medicine in China.

\section{DISCLOSURE}

In accordance with institutional policy, HS Warren has declared hemopexin to Massachusetts General Hospital as a potential candidate molecule to help decrease inflammation, and the institution has filed for patent protection. M Super, AL Watters and DE Ingber have filed for patent protection for recombinant Fc-hemopexin.

\section{REFERENCES}

1. Hebert PC, et al. (1999) A multicenter, randomized, controlled clinical trial of transfusion requirements in critical care. Transfusion Requirements in Critical Care Investigators, Canadian Critical Care Trials Group. N. Engl. J. Med. 340:409-17.

2. Malone DL, Dunne J, Tracy JK, Putnam AT, Scalea TM, Napolitano LM. (2003) Blood transfusion, independent of shock severity, is associated with worse outcome in trauma. J. Trauma. 54:898-905.

3. Lacroix J, et al. (2007) Transfusion strategies for patients in pediatric intensive care units. N. Engl. J. Med. 356:1609-19.

4. Reeves BC, Murphy GJ. (2008) Increased mortality, morbidity, and cost associated with red blood cell transfusion after cardiac surgery. Curr. Opin. Cardiol. 23:607-12.

5. Adamson JW. (2008) New blood, old blood, or no blood? N. Engl. J. Med. 358:1295-6.

6. Spinella PC, et al. (2009) Duration of red blood cell storage is associated with increased incidence of deep vein thrombosis and in hospital mortality in patients with traumatic injuries. Crit. Care. 13:R151.

7. Eikelboom JW, Cook RJ, Liu Y, Heddle NM. (2010) Duration of red cell storage before transfusion and in-hospital mortality. Am. Heart J. 159:737-43.

8. Pettila V, et al. (2011) Age of red blood cells and mortality in the critically ill. Crit. Care. 15:R116.

9. Aubron C, Nichol A, Cooper DJ, Bellomo R. (2013) Age of red blood cells and transfusion in critically ill patients. Ann. Intensive Care. 3:2.

10. Sowemimo-Coker SO. (2002) Red blood cell hemolysis during processing. Transfus. Med. Rev. 16:46-60.

11. Berra L, et al. (2012) Transfusion of stored autologous blood does not alter reactive hyperemia index in healthy volunteers. Anesthesiology. 117:56-63.

12. Lin T, et al. (2010) Synergistic inflammation is induced by blood degradation products with microbial Toll-like receptor agonists and is blocked by hemopexin. J. Infect. Dis. 202:624-32.

13. Fernandez PL, et al. (2010) Heme amplifies the innate immune response to microbial molecules through spleen tyrosine kinase (Syk)-dependent reactive oxygen species generation. J. Biol. Chem. 285:32844-51.

14. Lin T, et al. (2012) Identification of hemopexin as an anti-inflammatory factor that inhibits synergy of hemoglobin with HMGB1 in sterile and infectious inflammation. J. Immunol. 189:2017-22. 
15. Vinchi F, et al. (2013) Hemopexin therapy improves cardiovascular function by preventing hemeinduced endothelial toxicity in mouse models of hemolytic diseases. Circulation. 127:1317-29.

16. Larsen $\mathrm{R}$, et al. (2010) A central role for free heme in the pathogenesis of severe sepsis. Sci. Transl. Med. 2:51ra71.

17. Adamzik M, Hamburger T, Petrat F, Peters J, de Groot H, Hartmann M. (2012) Free hemoglobin concentration in severe sepsis: methods of measurement and prediction of outcome. Crit Care. 16:R125.

18. Janz DR, et al. (2013) Association between cellfree hemoglobin, acetaminophen, and mortality in patients with sepsis: an observational study. Crit. Care Med. 41:784-90.

19. Paoli M, Anderson BF, Baker HM, Morgan WT, Smith A, Baker EN. (1999) Crystal structure of hemopexin reveals a novel high-affinity heme site formed between two beta-propeller domains. Nat. Struct. Biol. 6:926-31.

20. Tolosano E, Altruda F. (2002) Hemopexin: structure, function, and regulation. DNA Cell. Biol. 21:297-306.

21. Hrkal Z, Vodrazka Z, Kalousek I. (1974) Transfer of heme from ferrihemoglobin and ferrihemoglobin isolated chains to hemopexin. Eur. J. Biochem. 43:73-8.

22. Cheung PK, Klok PA, Baller JF, Bakker WW. (2000) Induction of experimental proteinuria in vivo following infusion of human plasma hemopexin. Kidney Int. 57:1512-20.

23. Bakker WW, et al. (2005) Altered activity of plasma hemopexin in patients with minimal change disease in relapse. Pediatr. Nephrol. 20:1410-5.

24. Bakker WW, et al. (2005) Protease activity of plasma hemopexin. Kidney Int. 68:603-10.

25. Spiller F, et al. (2011) Inhibition of neutrophil migration by hemopexin leads to increased mortality due to sepsis in mice. Am. J. Respir. Crit. Care Med. 183:922-31.

26. Mauk MR, Smith A, Mauk AG. (2011) An alternative view of the proposed alternative activities of hemopexin. Protein Sci. 20:791-805.

27. Brinkman N, inventor; CSL Behring LLC, assignee. Method of purifying proteins. United States patent pub. no. US 2014/0094411 A1. 2014 Apr 3.

28. Smith A, Morgan WT (1984) Hemopexinmediated heme uptake by liver. Characterization of the interaction of heme-hemopexin with isolated rabbit liver plasma membranes. J. Biol. Chem. 259:12049-53.

29. Figueiredo RT, et al. (2007) Characterization of heme as activator of Toll-like receptor 4. J. Biol. Chem. 282:20221-9.

30. Satoh T, Satoh H, Iwahara S, Hrkal Z, Peyton DH, Muller-Eberhard U. (1994) Roles of heme iron-coordinating histidine residues of human hemopexin expressed in baculovirus-infected insect cells. Proc. Natl. Acad. Sci. U.S.A. 91:8423-7.

31. Warren HS, et al. (1993) Assessment of ability of murine and human anti-lipid A monoclonal antibodies to bind and neutralize lipopolysaccharide. J. Exp. Med. 177:89-97.
32. Marie C, Pitton C, Fitting C, Cavaillon JM. (1996) IL-10 and IL-4 synergize with TNF-alpha to induce IL-1ra production by human neutrophils. Cytokine. 8:147-51.

33. Rankin JA, Sylvester I, Smith S, Yoshimura T, Leonard EJ. (1990) Macrophages cultured in vitro release leukotriene B4 and neutrophil attractant/ activation protein (interleukin 8) sequentially in response to stimulation with lipopolysaccharide and zymosan. J. Clin. Invest. 86:1556-64.

34. Monteiro AP, et al. (2011) Leukotriene B4 mediates neutrophil migration induced by heme. J. Immunol. 186:6562-7.

35. Ashbaugh DG, Bigelow DB, Petty TL, Levine BE. (1967) Acute respiratory distress in adults. Lancet. 2:319-23.

36. Bastarache JA, et al. (2012) Low levels of tissue factor lead to alveolar haemorrhage, potentiating murine acute lung injury and oxidative stress. Thorax. 67:1032-9.

37. Schaer DJ, Buehler PW. (2013) Cell-free hemoglobin and its scavenger proteins: new disease models leading the way to targeted therapies. Cold Spring Harb Perspect Med. 3.

38. Mumby S, Ramakrishnan L, Evans TW, Griffiths MJ, Quinlan GJ. (2014) Methemoglobin-induced signaling and chemokine responses in human alveolar epithelial cells. Am. J. Physiol. Lung Cell. Mol. Physiol. 306:L88-100.

39. Pamplona A, et al. (2007) Heme oxygenase-1 and carbon monoxide suppress the pathogenesis of experimental cerebral malaria. Nat. Med. 13:703-10.

40. Chung SW, Liu X, Macias AA, Baron RM, Perrella MA. (2008) Heme oxygenase-1-derived carbon monoxide enhances the host defense response to microbial sepsis in mice. J. Clin. Invest. 118:239-47.

Cite this article as: Lin T, et al. (2016) Purified and recombinant hemopexin: protease activity and effect on neutrophil chemotaxis. Mol. Med. 22:22-31. 